\title{
contrôle des mouvements de surface lors de travaux souterrains urbains
}

par

M. Viallaneix

R. A. T.P.-N.S. 1

RESUME - La construction d'ouvrages souterrains en site urbain dans des terrains difficiles impose de mettre en oeuvre des méthodes d'exécution limitant au raximum la décompression des terrains et par conséquent les tassements en surface. Ces tassements, très préjudiciables à la stabilité des immeubles, doivent être parfaitement contrôlés, au cours de l'exécution des travaux, par auscultation d'un ensemble de renères topooraohiques.

Lẹs résultats obtenus, par cette méthode très rapide, permettent éventuellement d'adapter ou de corriger la méthode d'exécution initialement retenue et d'apporter in fine, les éléments nếcessaires à la programmation des interventions complémentaires dans les zones les plus perturbées.

\section{INTRODUCTION}

La demande toujours croissante de moyens de transport efficaces dans les grandes agơlomérations conduit à la réalisation de nombreux tunnels de métro. Ces travaux sont très souvent réalisés dans des zones fortement urbanisées où le recours à des méthodes de construction à ciel ouvert est impossible. On est alors conduit à construire des tunnels à l'avancement par des méthodes purement souterraines qui peuvent s'accompagner de tassements de terrains très préjudiciables aux bâtiments implantés. C'est pourquoi il est nécessaire de procéder en cours de travaux à une surveillance topographique afin de prévenir les désordres éventuels.

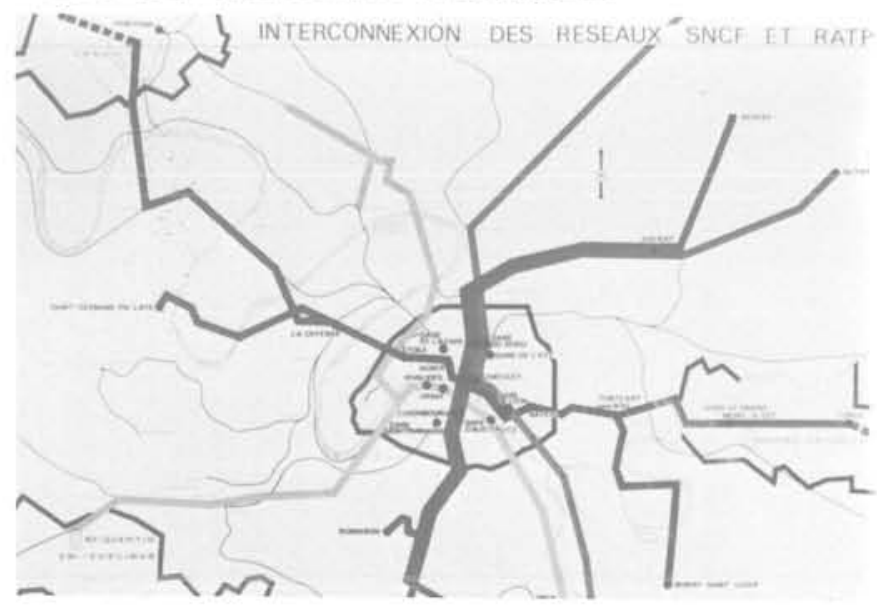

Fig. 1 - Plan de l'interconnexion
Au travers d'un chantier réalisé dans Paris, l'exposé se propose de montrer l'intérêt des mesures de nivellement de surface.

\section{PRESENTATION DES OUVRAGES A CONSTRUIRE}

\section{Situation géographique}

Les travaux présentés s'insèrent dans le programme d'interconnexion des réseaux SNCF et RATP dans Paris, conçus pour des trains à gabarit conforme aux normes internationales (UIC). (Fig. 1).

Ils concernent plus particulièrement le prolongement de la ligne B du RER de "Châtelet Les Halles" à "Gare du Nord", jonction qui verra fin 1981 la naissance d'une ligne régionale Nord Sud transitant par "Châtelet Les Halles" - (Fig. 2).

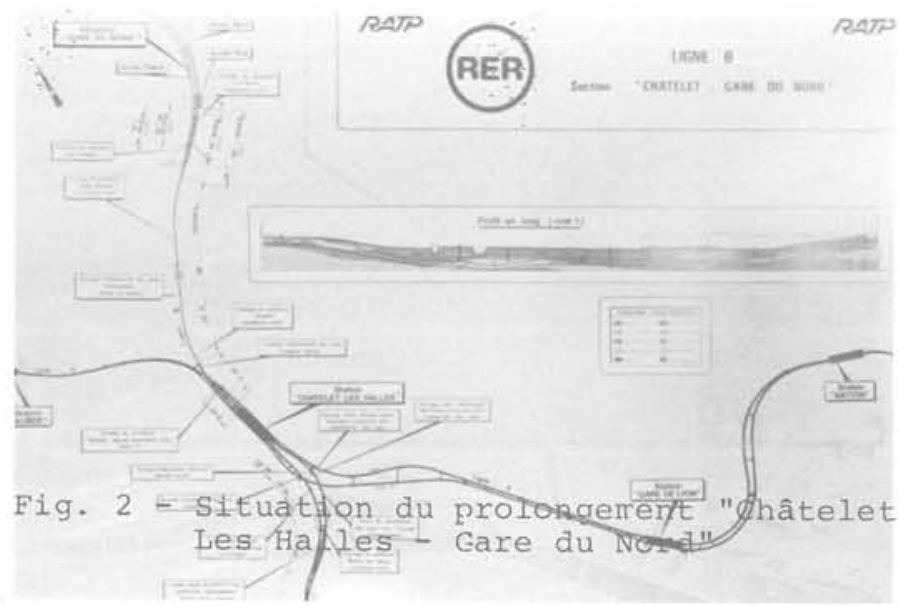


Le prolongement d'environ $2.500 \mathrm{~m}$ de longueur, se décompose en 3 lots géographiques distincts : (Fig. 3).

- les ouvrages d'arrière gare de "Châtelet Les Halles",

- un souterrain à 2 voies de $9,10 \mathrm{~m}$ d'ouverture et de $1.250 \mathrm{~m}$ de longueur,

- les ouvrages spéciaux d'arrivée à "Gare du Nord".

La totalité de ces ouvrages se développe en permanence sous un tissu urbain très dense constitué d'immeubles de 5 à 6 étages de construction ancienne. Ils se trouvent entre 5 et $25 \mathrm{~m}$ de profondeur et se faufilent entre de nombreux ouvrages existants (Fig. 5).

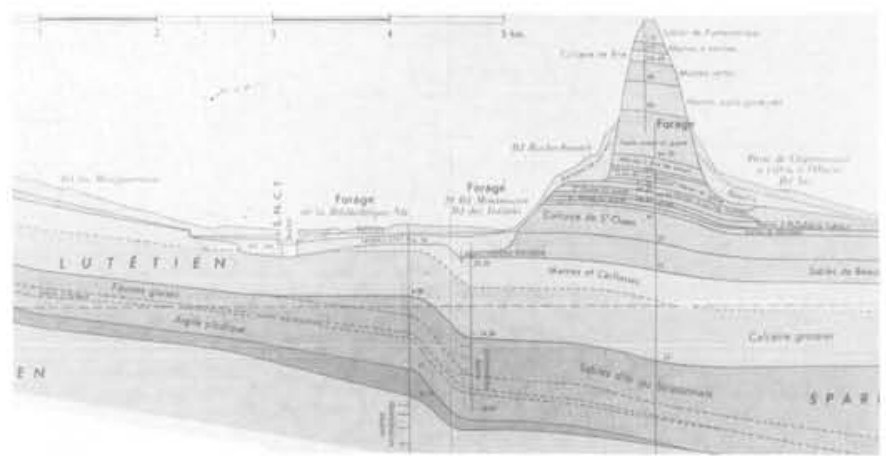

Fig. 4 - Profil en long de la géologie du bassin parisien

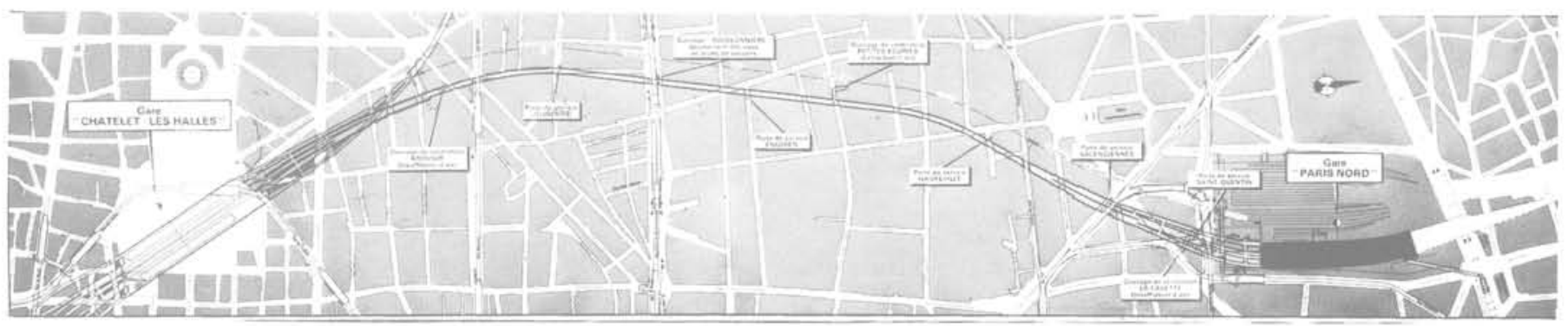

Fig. 3 - Vue en plan du prolongement "Châtelet - Les Halles - Gare du Nord"

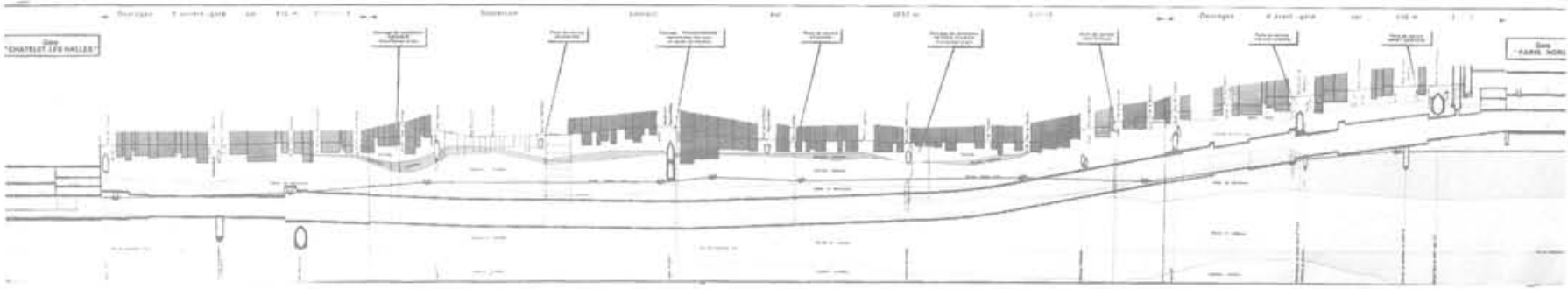

Fig. 5 - Profil en long "Châtelet - Les Halles - Gare du Nord"

\section{Géologie des terrains rencontrés}

La majeure partie du projet évolue dans la plaine alluviale de la seine où nous retrouvons la géologie classique du bassin parisien comprenant successivement des remblais, des alluvions, des sables de Beauchamps, des marnes et caillasses (Fig. 4).

Le tunnel à construire se situe essentiellement dans ce dernier horizon (Fig. 5).

Toutefois, dans ce secteur particulier, l'horizon géologique des marnes et caillasses comporte un dépôt de gypse dont l'épaisseur crôt vers le Nord-Est de Paris.

Le caractère soluble du gypse inclus dans cette formation, qui est baignée par une nappe aquifère très active, a amené, au cours des temps géologiques, de profondes désorqanisations dans la structure des marnes et caillasses. Sa dissolution a conduit à la formation de vides francs, de zones décom- primées ou de vides remblayés par déversement des horizons supérieurs pouvant se traduire au stade ultime par création de fontis progressant vers la surface (Fig. 6).

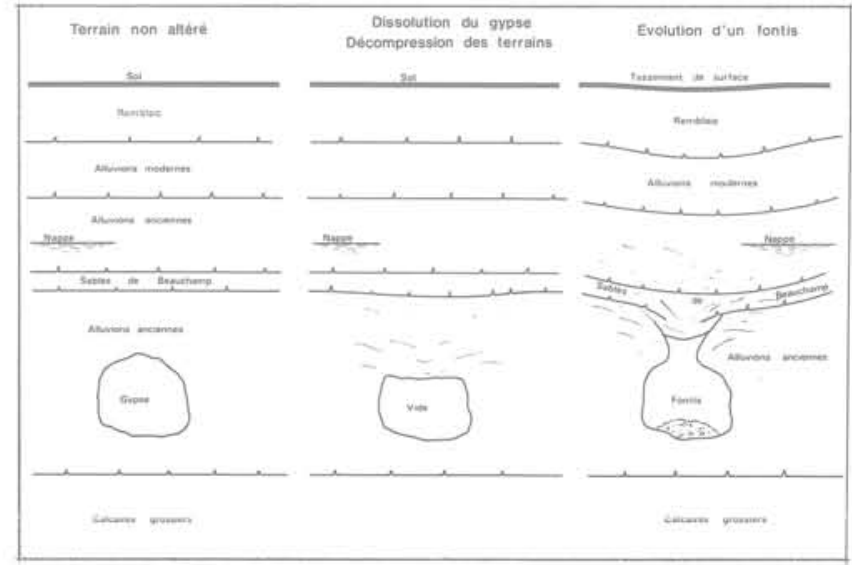

Fig. 6 - Résultat de la dissolution du gypse 
Méthode d'exécution adoptée

La dernière partie de l'exposé s'appuyant sur un secteur très limité du tunnel courant, seule la méthode d'exécution de celui-ci sera abordée.

Le niveau de difficultés exceptionnelles à conduit la RATP à mettre en oeuvre une méthode d'exécution permettant de s'adapter aux conditions locales.

Ainsi, la réalisation du souterrain a comporté quatre phases successives :

- Creusement d'une galerie pilote. Implantée au coeur de la section, cette galerie a permis une reconnaissance à l'échelle réelle, de la géologie de détail, sur toute la longueur du lot. Des anomalies de type fontis, vides, poches d'alluvions ont été localisées avec précision.

- Exécution de traitement de terrain. Grâce à la galerie pilote la reconnaissance continue a servi à déterminer, zone par zone, le type de traitement. On a ainsi réalisé un traitement sélectif, adapté aux conditions locales, dont l'épaisseur varie de $1,30 \mathrm{~m}$ dans les zones les plus saines à $2,50 \mathrm{~m}$ dans les zones perturbées (Fig. 7, coupe BB).

- Réalisation de la voûte (Fig. 7). Le procédé utilisé, le prédécoupage mécanique, consiste à exécuter le terrassement de la demi-section supérieure, à l'abri d'une prévoute réalisée préalablement par prédécoupage d'une saignée de faible épaisseur suivant 1 'extrados de la voute et par son remplissage à l'aide de béton projeté. La voûte est ensuite bétonnée par anneau de $4,00 \mathrm{~m}$ à une distance de $15 \mathrm{~m}$ du front de taille. Outre la possibilité de mécanisation très poussée du terrassement, ce procédé permet d'établir une coque résistante avant l'excavation et de gagner de vitesse les déformations inévitables des terrains.

En réduisant la longueur du terrassement, donc en augmentant le recouvrement des prévoûtes, cette méthode a l'avantage de permettre le passage de zones particulièrement délicates (franchissement d'ouvrage, zone de terrains instables).

Exécution de la demi-section inférieure (Fig. 8).

Afin de limiter les tassements de la voûte sur ces appuis provisoires, notamment dans les zones où les terrains sont fortement décomprimés, l'execution de la demi-section inférieure suit la voûte avec un décalage minimum.

Le procédé consiste à réaliser par reprise en sous oeuvre de la voûte, des plots de piédroits de 4,50 m de lonģueur, le bétonnage du radier suivant immédiatement I'exécution des piédroits.

La mise en oeuvre de cette méthode d'exécution étudiée de manière très minutieuse, était dictée par la nécessité d'une réalisation rapide des ouvrages dans un programe très tendu, mais aussi par le souci permanent de limiter au minimum la décompression des sols et par conséquent des mouvements en surface.

Néanmoins, du fait d'une aéologie très tourmentée, des décompressions n'ont pu être évitées dans certaines zones très sensibles.

Ainsi, parallèlement à l'exécution du souterrain, une surveillance de ces mouvements s'est imposée.

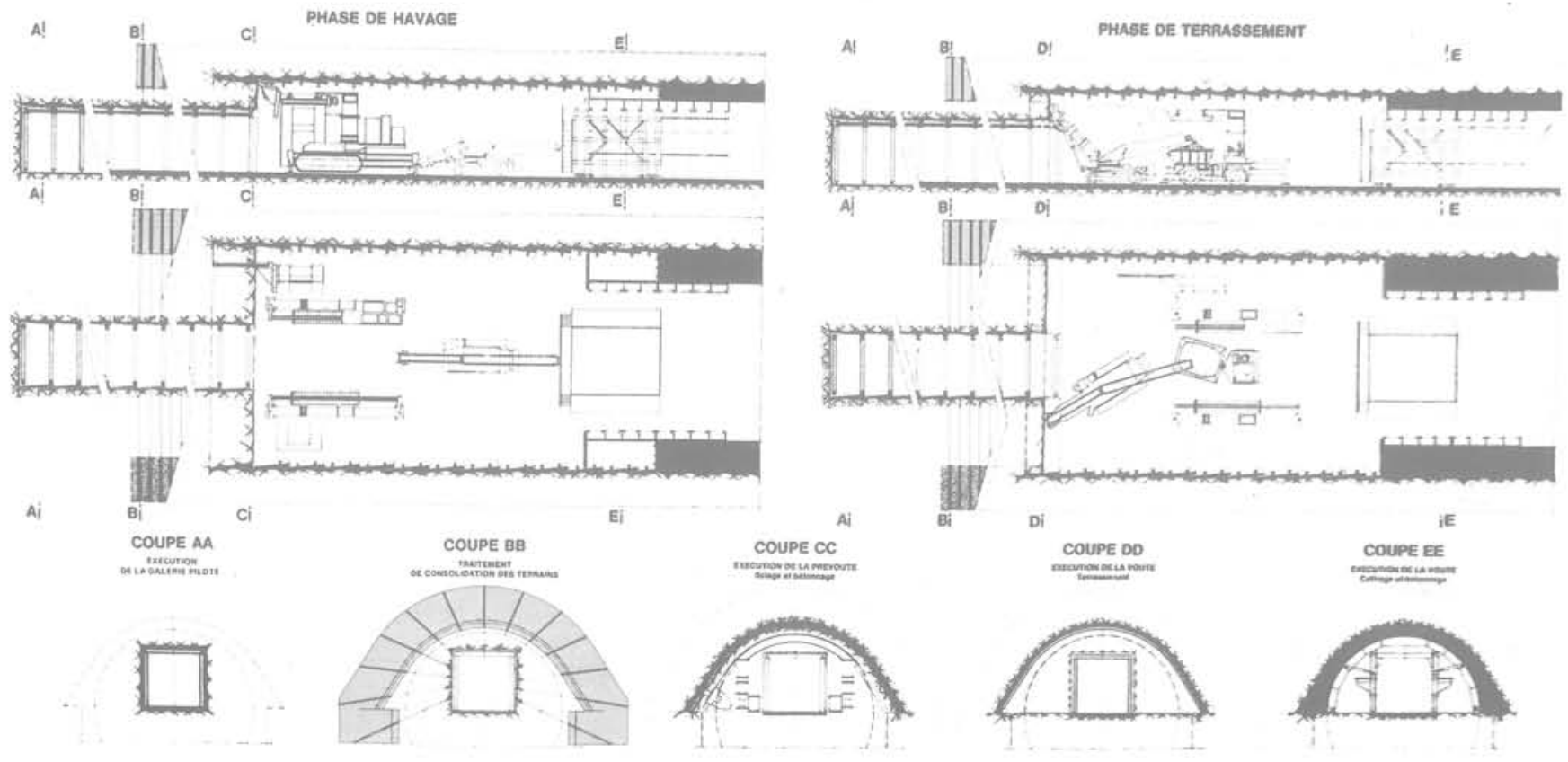

Fig. 7 - Méthode d'exécution de la demi-section supérieure 


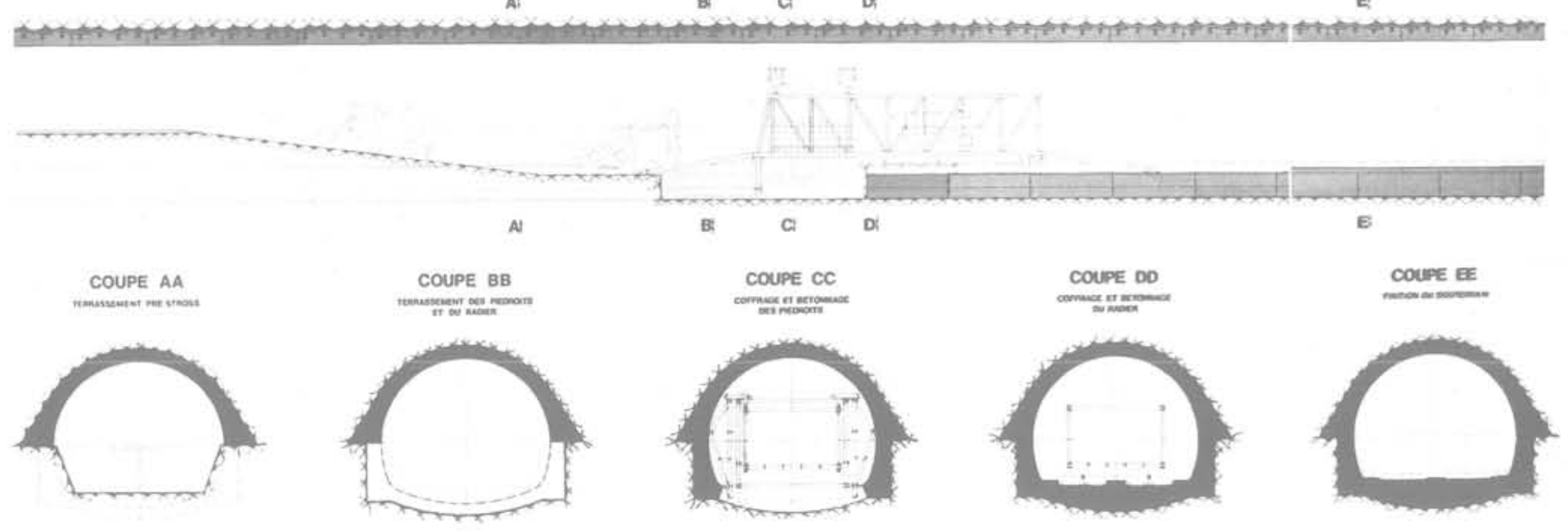

Fig. 8 - Méthode d'exécution de la demi-section inférieure

\section{CONTROLE DES MOUVEMENTS DE TERRAINS}

\section{Les repères}

La constatation du tassement est réalisée par auscultation topographique de repères collếs sur les immeubles. Ces repères sont constitués de plaquettes d'acier munies d'une bille support, de faible diamètre, sur laquelle reposera la mire. Ils sont implantés en bordure des immeubles ou dans les cours intérieures et intéressent une bande de terrain de l'ordre de $80 \mathrm{~m}$ de part et d'autre de I'axe du tunnel (Fig. 9). La densité des points est d'environ 20 points à l'hectomètre de tunnel.

Le réseau de nivellement (Fig. 9)

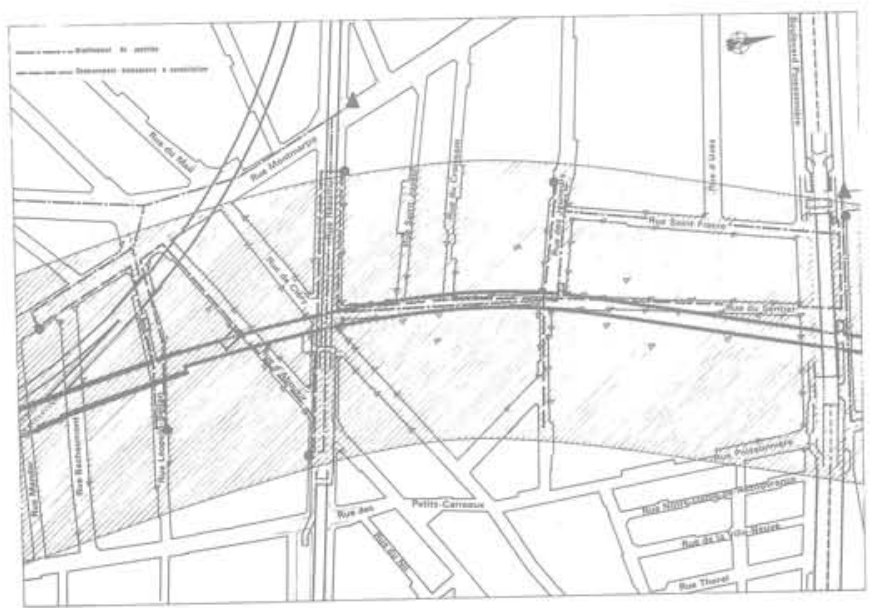

Fig. 9 - Réseau d'auscultation en surface

Les repères d'auscultation sont reliés au réseau de nivellement général par l'intermédiaire de points secondaires situés hors de la zone en mouvement. Les points sont considérés comme insensibles aux fluctuations du chantier et servent de fermeture aux cheminements élémentaires d'auscultation.
De cette façon, il peut être facilement procédé à des mesures de périođicité variable selon l'avancement des travaux puisque chaque cheminement élémentaire, de $300 \mathrm{~m}$ de longueur environ, peut être effectué indépendamment.

Le nivellement de jonction d'environ $3 \mathrm{~km}$ de longueur est effectué avant tout début des travaux et contrôlé tous les 6 mois environ.

\section{RESULTATS D'AUSCULTATION}

Chaque noint est surveillé par la méthode du cheminement double (Cholesky). La précision du nivellement pour un repère de tassement est d'ordre de $5 / 10$ e de $\mathrm{mm}$.

L'exploitation de ces résultats par le constructeur nécessite l'élaboration de documents de synthèse.

Courbe d'égal tassement ou isolapses (Fig.10)

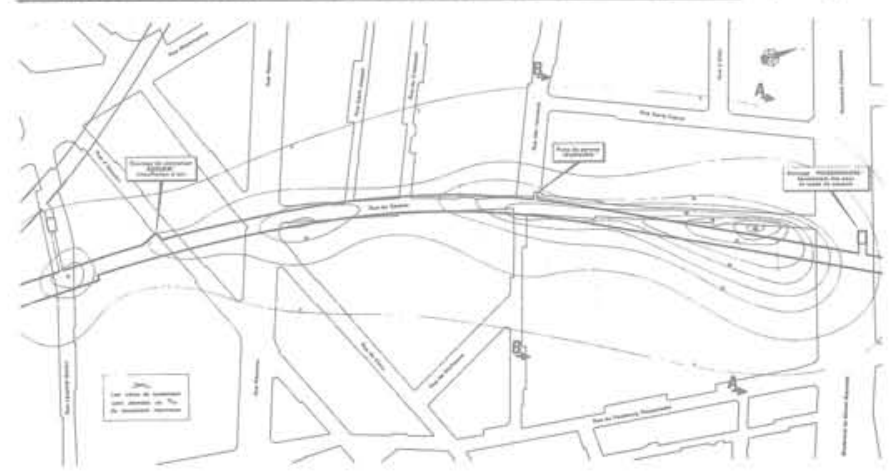

Fig. 10 - Isolapses

Les courbes d'ẹcal tassement ou isolanses, dont 1'utilisation est frécuente dans les régions minières, nermettent d'obtenir une vue d'enserble sur les tassements constatés et définissent parfaitement en plan la zone d'influence des travaux. Elles permettent par ailleurs, de connaître en tout point, i'importance du tassement différentiel. 
Dans le cas précis de ce chantier, le suivi de l'évolution des isolapses pour les tassements engendrés, par le creusement de la galerie pilote, a permis d'apoorter un élément complémentaire à la reconnaissance des terrains. C'est ainsi que dans les zones sensibles le schéma de traitement adapté tient compte de ces résultats.

Il est à noter la largeur exceptionnelle de la surface intéressée par les mouvements de terrains.

Profil lonqitudinal des tassements (Fig. 11)

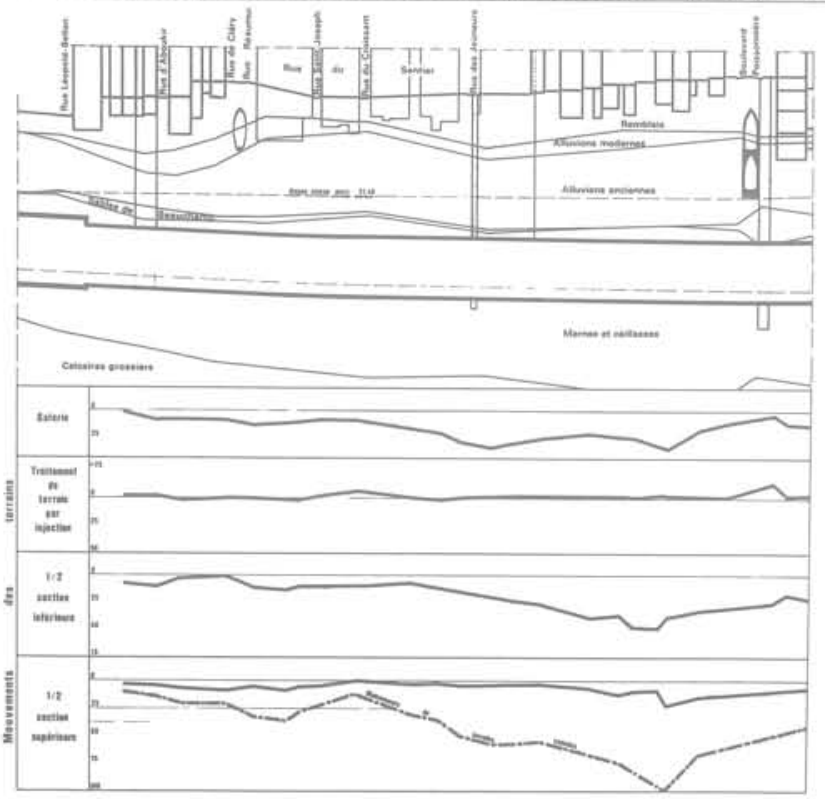

Fig. 11 - Profil en long des mouvements de terrain phase par phase

Le profil longitudinal de tassement montre, pour un instant donné l'importance du tassement en fonction de l'avancement du chantier et des terrains rencontrés.

Ainsi le tassement moyen, provoqué par chaque phase de travail est en moyenne de :

- 35 \& pour 1- galerie pilote $(S=0,15 . S T)$

- 40 \% pour la demi-section supérieure $(\mathrm{S}=0,40 . \mathrm{ST})$

- 25 \% pour la demi-section inférieure $(\mathrm{S}=0,45 . \mathrm{ST})$

ST : section totale de la section excavée.

On remarquera l'importance du tassement dû à la galerie pilote pour une section excavée d'environ 15 \% de la section totale.

Profil transversal de tassement (Fig. 12) La zone d'influence de l'excavation, transversalement au tunnel, peut être constatée par des profils transversaux. Ce type de représentation permet d'analyser pour un même immeuble, les risques de tassement différentiels qui occasionnent des effets très préjudiciables à la stabilité des constructions et font apparaitre des fissures dans les murs porteurs.

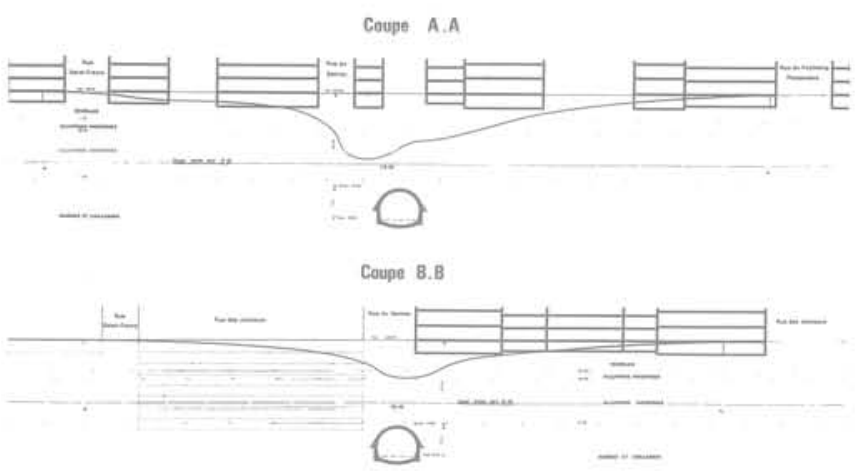

Fig. 12 - Profil en travers des mouvements de terrain

Le profil transversal des tassements permet aussi de calculer les rayons de courbure de la cuvette et de déterriner en suivant son évolution, le rayon critique caractérisé par l'apparition des premiers désordres (Fig.13).

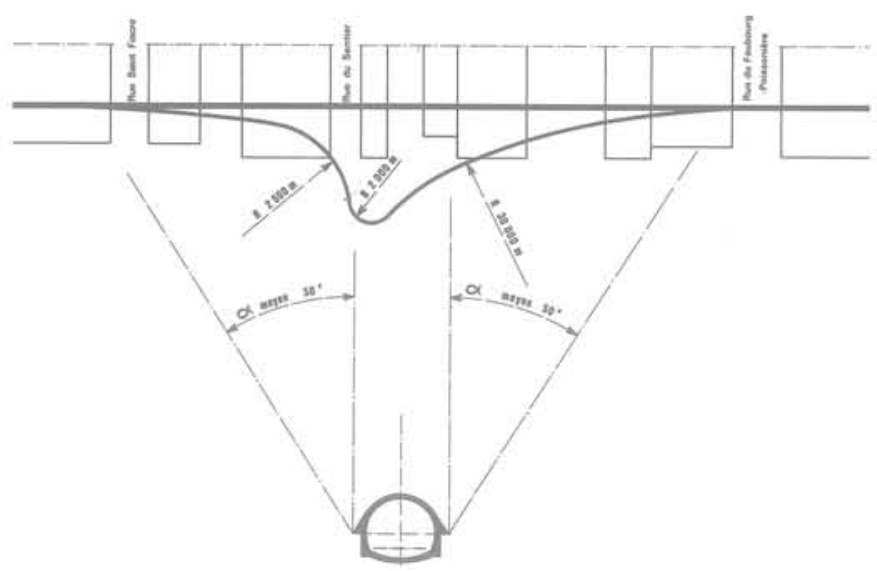

Fig. 13 - Ançle d'influence - Rayons de courbure

Les courbes apnellent un certain nombre de remarques. Tout d'abord, et contrairement au phénomène de tassement habituel, le mouvement maximum ne se produit pas systématiquement à la verticale de l'excavation, ce qui dénote l'importance de la nature des terrains dans le processus d'affaissement. Le phénomène trouve son amplitude maximum dans les zones les olus perturbées. Par ailleurs, il apparâ̂t que les désordres les plus importants sur les imreubles se produisent dans certains cas, dans des secteurs où le rayon de courbure est très grand, ce qui montre que la forme des bâtiments est fondamentale et en particulier le rapport hauteur sur largeur. 
Courbe de mouvement d'un point (Fig. 14)
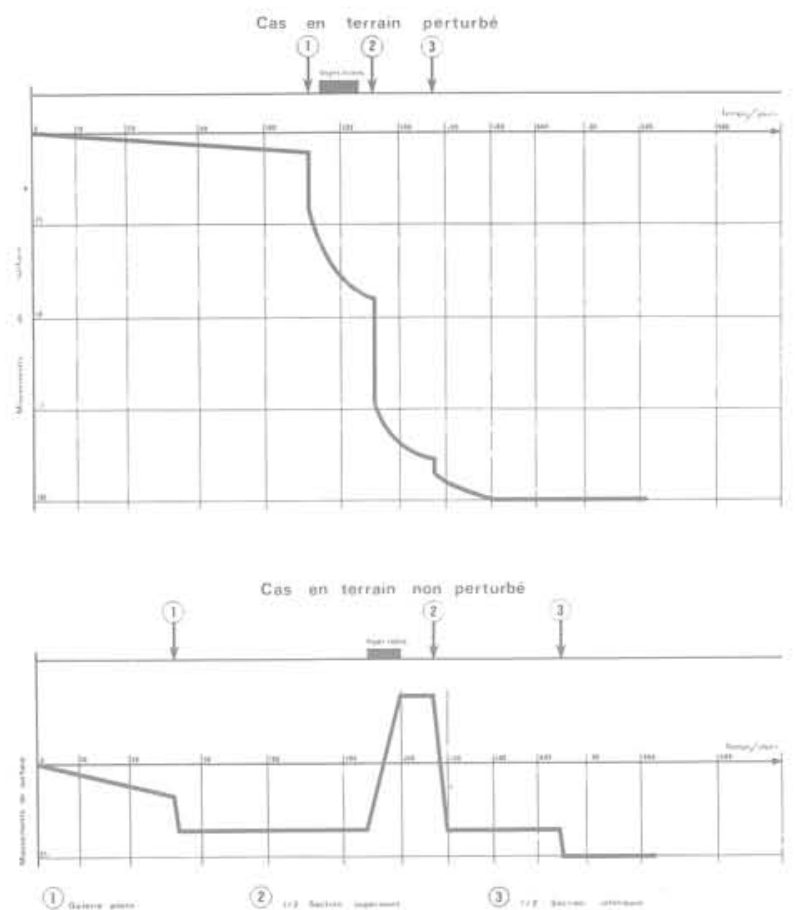

Fig. 14 - Evolution d'un point

Un graphique précisant l'évolution des mouvements en fonction du temps, document de base du nivellement, caractérise chaque point. Par comparaison des graphiques de différents points situés au-dessus d'horizons géologiques différents, la réaction du sol selon les terrains rencontrés, peut en particulier être mise en évidence.

Ainsi on remarquera que les zones de terrains perturbés, outre une amplitude plus importante, sont le siège de tassements différés provenant vraisemblablement d'une recompression des horizons décomprimés.

Par ailleurs, le repérage des principales étapes de 1'avancement des travaux à 1'aplomb du point considéré fournit de précieuses indications relatives à la progression des mouvements en fonction des phases de travaux.

\section{INTERETS DES CONTROLES DE MOUVEMENTS DE TERRAINS}

Ainsi que nous venons de le voir, les mesures de tassements permettent l'établissement d'un certain nombre de graphiques susceptibles d'apporter par leur analyse, quelques enseignements sur le comportement des terrains et sur la valeur des méthodes d'exécution, mais aussi d'optimiser le choix des interventions complémentaires à effectuer dans les zones où les désordres sont les plus importants.
Ainsi, en fin de chantier il est possible d'élaborer le document précisé fig. 15.

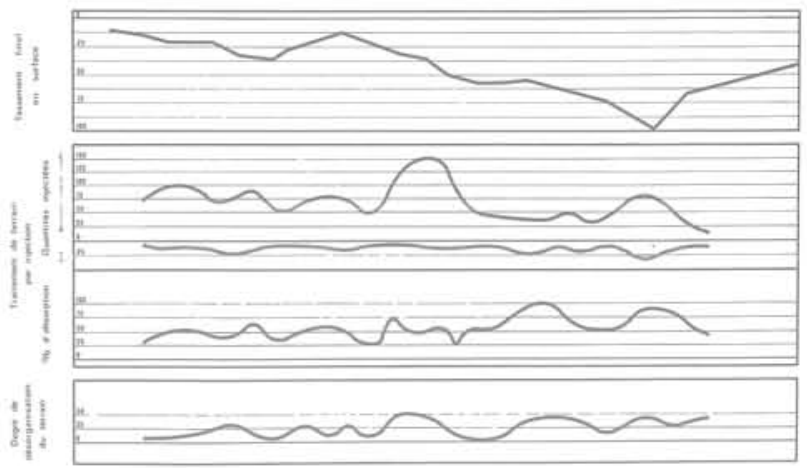

Fig. 15 - Relation tassements - Volumes injectés - o d'absorotion - Degrés de désorganisation des terrains traversés

Outre, le profil longitudinal des mouvements, ce type de document précise parallèlement les quantités réelles injectées, le ò d'absorption et le degré de désorananisation du terrain (surface d'alluvions d'un front donné rapportée à la surface totale de la section).

Ce type de docurent est très précieux pour réaliser oar exemnle des traitements de compensation sous les immeubles ayant subi des tassements imnortants. Il permet une étude précise du projet éventuel.

Au niveau de l'établissement d'un projet futur, il est intéressant d'utiliser les résultats antérieurs. Par exemole la connaissance de l'angle d'influence probable facilite la recherche du tracé optimal vis-à-vis des immeubles et les notions d'influence des facteurs géologiques apportent des éléments indispensables au choix du profil en long du tunnel.

\section{CONCLUSION}

Les mesures de tassements, outre leur rôle de constatation, offrent des renseignements substantiels susceptibles de faire progresser la connaissance des facteurs liés au creusement des souterrains.

L'interprétation des résultats permet, en expliquant certains phénomènes, d'améliorer et d'adapter les méthodes d'exécution et constitue aussi un élément complémentaire des différents paramètres tels que la géologie, la géotechnique et l'hydrologie.

Mais, il faut néanmoins noter que l'analyse des tassements conduit à des constatations, voire à des interprétations a posteriori et en aucun cas la mesure de ceux-ci ne peut stopper la progression du phénomène. 